\title{
Compression Image by Using Iterated Function Systems
}

\section{Basil Y. Al-khayat \\ Mahasin Thabit Younis \\ mahasinthabit@uomosul.edu.iq \\ College of Computers Sciences and mathematics \\ University of Mosul}

\section{Received on: 30/5/2011}

Accepted on: 2/11/2011

\section{ABSTRACT}

This research was in the compression digital images using the iterative function and applied to grayscale images, color images and then re-pressed image decoding. Matlab language is used for programming the system, the proposed algorithms have been applied to a number of images and the results show the efficient and accurate of the proposed algorithms. The method of compressing using iterative function systems methods are from loss compressing methods the advantage of these method by high compressing rate and this was confirmed by the results obtained have been getting a good percentage of the compressing and the quality is good and clear.

Keywords: Fractal Compression, Iterated Function Systems, Image Processing.

$$
\begin{aligned}
& \text { كبس الصور الرقمية باستخدام انظمة الدالة التكرارية }
\end{aligned}
$$

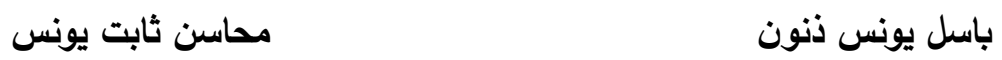

$$
\begin{aligned}
& \text { كلية علوم الحاسوب والرياضيات، جامعة الدوصل لبن }
\end{aligned}
$$

تاريخ قبول البحث: 2011/11/02

تاريخ استلام البحث: 2011/05/30

الملخص

تم في هذا البحث كبس الصور الرقمية باستخدام الدالة التكرارية وتطبيقها على صور ذات تدرج رمادي

وصور ملونة ومن ثم إعادة فك الصور المكبوسة. تم استخدام لغة Matlab لبرمجة النظام، تم تطبيق الخوارزميات

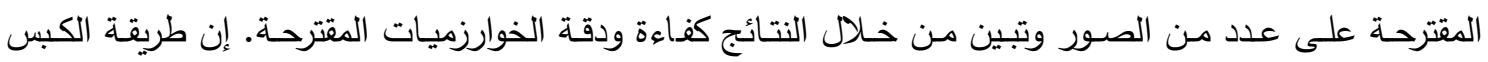

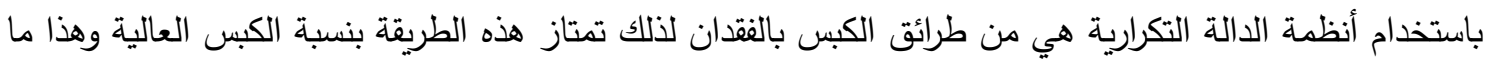
تم تأكيده مـن خـلال النتائج التي تم الحصول عليها حيث تم الحصول على على نسبة جيدة للكبس ونوعية جيدة وواضحة.

الكلمات المفتاحية: الكبس الكسوري، انظمة الدالة التكرارية، معالجة الصور •

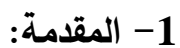

يُعد علم الكسوريات من العلوم الحديثة التي ظهرت في العقدين الأخيرين من القرن العشرين وهو علم تُمات

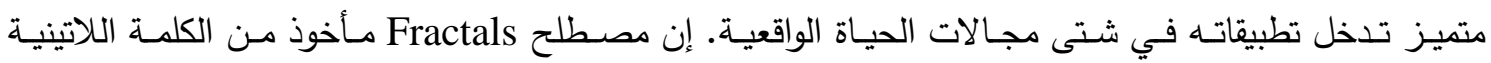

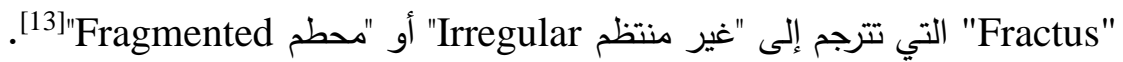
الكسوريات هي الأشكال التي تعرض التشابه الذاتي تحت أي مقياس من مقاييس التكبير ، ويمكن بناؤها بفكرة بسيطة (الصورة التي تتكرر على مقاييس صغيرة جداً).

أصبحت الكسوريات تشغل اهتمام الكثيرين منذ ظهور الحاسوب. تعرض الكثير من صور الطبيعة التثابه

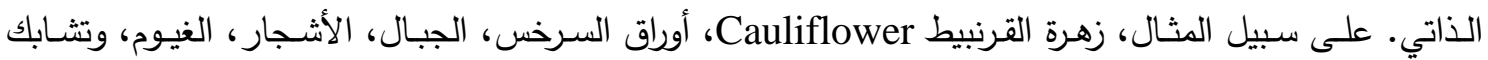
الأوعية الدموية في أجسامِنا، جميعها تمتلك بنية كسورية [10]ت.

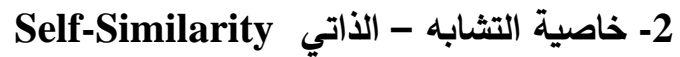


تُعد خاصية التثابه-الذاتي صفة مميزة للكسوريات Fractals، والتي تعني عشعشة لانهائية التهات Nesting من التراكيب على كل المقاييس التي تُشير إلى أنه عند عرض جزه ثات ثانوي من الشكل فإنه يُشتبه هيكلاً علوياً في الثكل نفسه].[3ن.

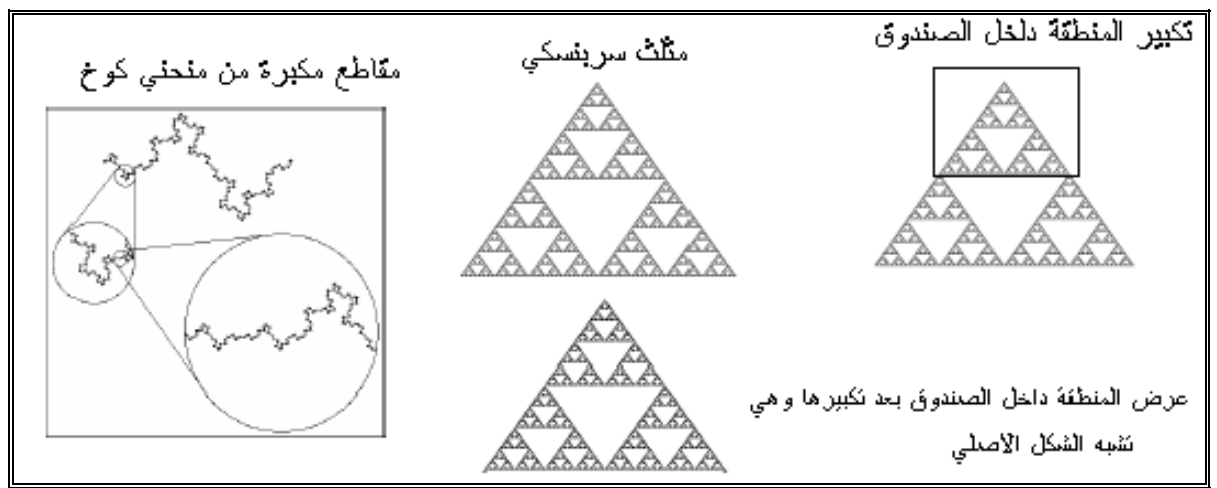

الثكل (1). خاصية التثابه-الذاتي لمقاطع مكبرة من أشكال كسورية.

3- كبس الصور الرقمية باستخدام أنظمة الدالة التكرارية

تُعد عملية إرسال البيانات وتخزينها من العمليات المكلفة. فكلما زادت كمية المعلومات التي تتعامل معها

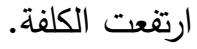

إن هدف كبس الصور الرقمية تقليل حجم ملف البيانات في الوقت الذي يتم فيه الاحتفاظ بالمعلومات

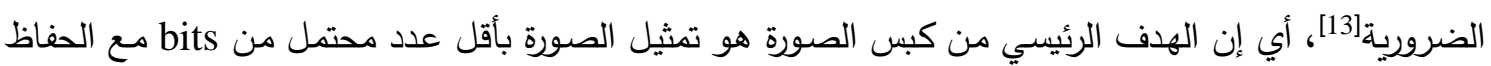
على مستوى الوضوح level of intelligibility [1]. هناك نوعان من طرائق الكبس وهما الكبس بدون فقد عندان 1. Run Length Code (RLC).

2. Huffman Coding.

3. Lemple Ziv Wett (LZW).

4. Arithmetic Coding. Lossless أما أشهر خوارزميات الكبس بفقدان فهي: 1. سويلات المويجة Wavelet Transform. 2. المكمم الاتجاهي 3. الشفرة التنبؤية Zerotree.

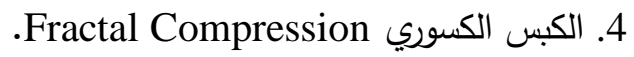
تعد أنظمة الدالة التكرارية Iterated Function Systems والتي يرمز لها اختصـارا بـ (IFS) الأساس وراء تطور مفهوم كبس الصور باستخدام الهندسة الكسورية إن الفكرة الأساس لأنظمة الدوال التكراريـة هي إنثاء

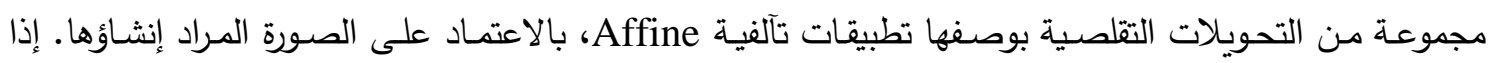

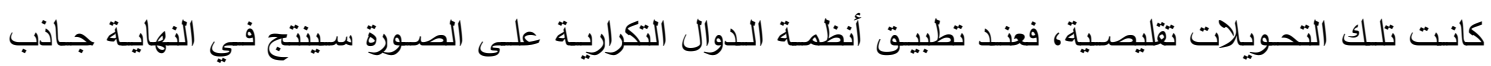
Attractor 
تبدأ طريقة الكبس باستخدام IFS بتقسيم الصورة إلى أجزاء تسمى كتل المدى range blocks بعدها

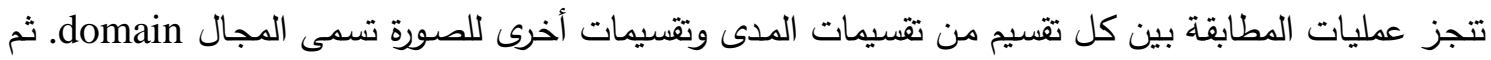

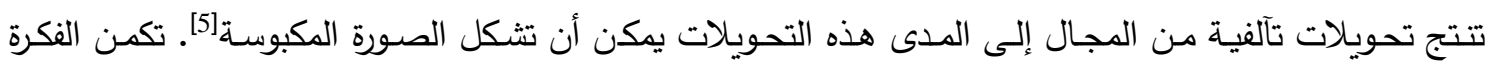
الرئيسة في تقسيم فضساء الصورة مرتين: مرة إلى كتل المدى التي تكون غير متداخلة مonoverlaping بحند

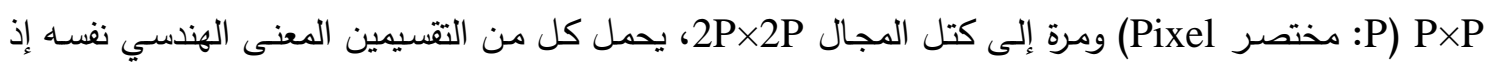
يغطيان الصورة الأصلية، ثم نقوم بإيجاد تطابق التحويل بين المدى والمجال بواسطة تحويلات محددة مثل: دوران، قلب، ... التخ. في النهايـة تم تعريف واحد من تحويلات التطبيق من خـلال متغيرين هما: القياس (scaling) والإزاحة (offset). أن عملية كبس الصورة تتم من خلال مطابقة كل كتلة من المدى بجميع كتل المجال لإيجاد

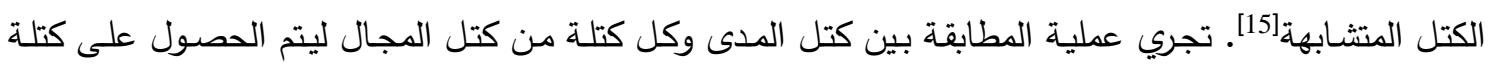
مجال مطابقة لكتلة المدى. إن الهدف من عملية المطابقة هي الحصول على أفضل كتلة مجال مطابقة لكتلة المدى باستخدام التحويلات التآلفية.

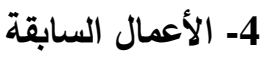

إن العديد من الطرائق التي استخدمت لمعالجة الصور الرقمية كانت تعتمد مفاهيم الهندسة الكسورية،

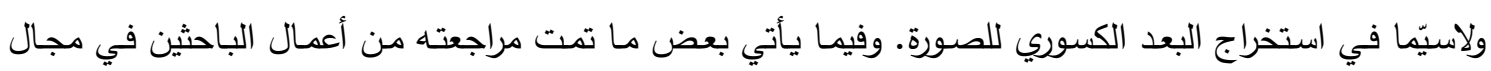

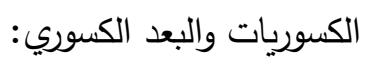

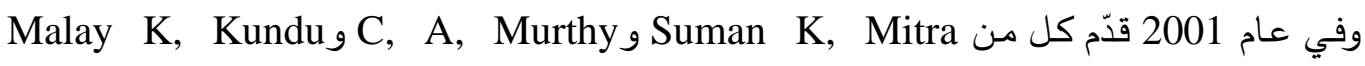

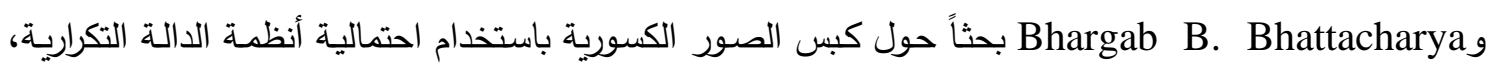
لإمكانية تعريف عوامل ماركوف المرافقة لقياس الاحتمالية المجزة للصورة المعطاة[114]. في عام 2004 قدّمت الباحثة إخلاص عبد الجبار الطائي رسالة ماجستير حول "تمييز الأشياء الطبيعية في الصـور الرقمية" مـن خـلال تحليل نسيج الصسورة باستخدام الهندسـة الكسورية وحسـاب البعد الكسوري لنسيج الصورة، ثم تقطيع الصورة بالاعتماد على قيم البعد الكسوري.

وفي العام نفسه قدمت الباحثة شيماء مضر يوسف النوري رسالة ماجستير حول "تقطيع الصور الرقمية باعتماد تحويل المويجة والكسوريات" لفهم مدى تأثير التحليل متعدد الدقة لتحويل المويجة على التقطيع الكسوري

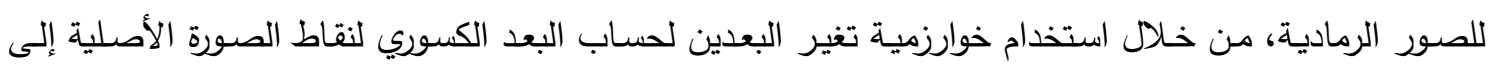

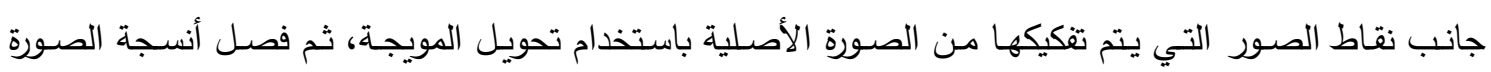
بالاعتماد على قيم البعد الكسوري التي تم حسابها ودراسة الفرق في النتائج •

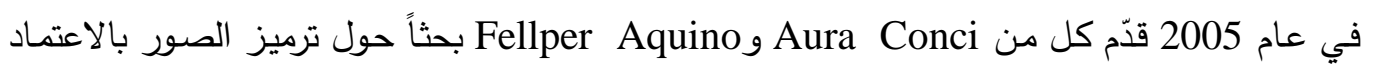
على البعد المحلي للصور الكسورية، وتصنيف الصورة بالاعتماد على البعد الكسوري وذلك لتقليل وقت الترميز [2]. 5- 5 التشابه الذاتي في الصور

في الثـكل (1) الذي يمثل صـورة لينـا لا نلاحظ أي نـوع مـن التشـابه الذاتي التي تتصف بهـا الكسوريات تحتوي على تحويلات مقاربة بالمعنى الحرفي. ولكن في الحقيقة هذه الصورة تحتوي على نوع مختلف من التثابه الذاتي. 


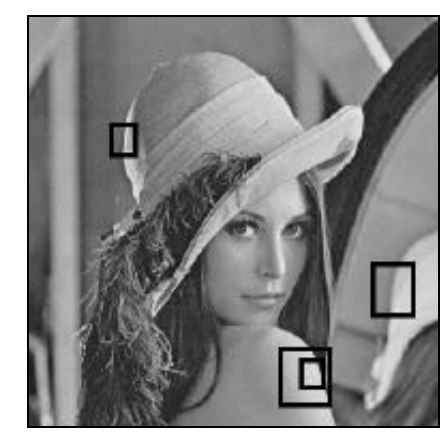

الثكل (2): التثابه الذاتي في صورة لينا.



الثكل (1): صورة Lena الأصلية

$(256 \times 256$ pixel $)$

الثكل (2) يظهر عينات لمناطق من صورة "لينا" متثـابهة عند مختلف المقاسـات: فجزء من الكتف

يحتوي على مناطق متداخلة تكون على الأغلب متشابهة، وكذلك فإن الجزء المنعكس على المرآة من القبعة مشابه (بعد التحويلات) لجزء من قبعتها. إن فرق هذا النوع من التشابه الذاتي هو أن الصورة بدل أن تُشَّكل من التكرار

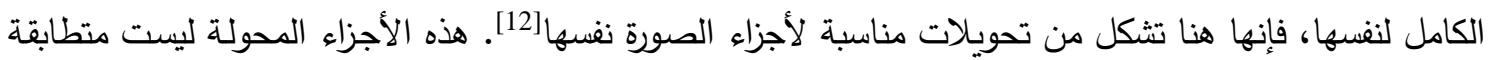

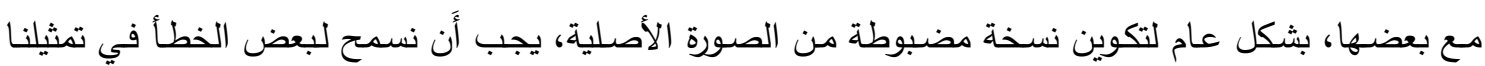
للصورة بمجموعة من التحويلات. هذا يعني أن الصورةً التي تمثل بمجموعة من التحويلات لن تكون نسخة مماثلة من الصورة الأصلية عند استرجاعها في عملية فلك الكبس لكن سوف تكون مقاربة لها [4] لهون.

\section{6- التحويلات التقلصية والتآلفية}

في الرسم الحاسوبي تبنى الصورة الثنائية البعد 2D جزءاً بعد جزء ثم يتم تحريرها بشكل مقنع، هذا

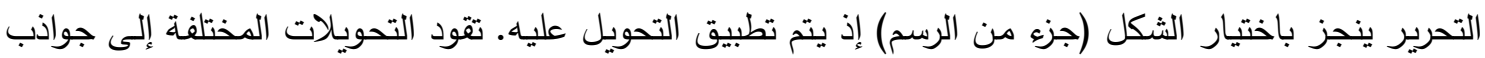

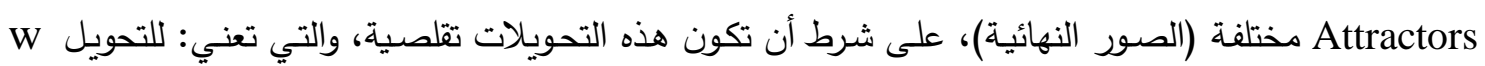
فإن أي نقطتين P و P P P P أن تكونا أكثر تقاربا في الصورة المستتخة عما هما عليه في الصورة الأصلية. إن

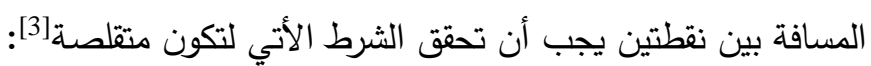

$\mathrm{d}\left(\mathrm{w}\left(\mathrm{P}_{1}\right), \mathrm{w}\left(\mathrm{P}_{2}\right)\right)<\mathrm{s} d\left(\mathrm{P}_{1}, \mathrm{P}_{2}\right)$ for $\mathrm{s}<1$.

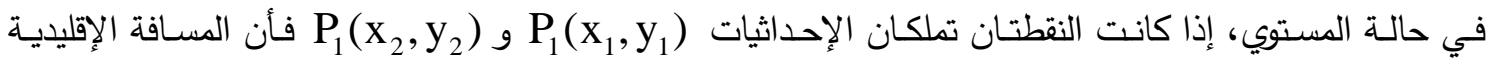
بينهما تحسب من العلاقة الآتية:

$\mathrm{d}\left(\mathrm{P}_{1}, \mathrm{P}_{2}\right)=\sqrt{\left(\mathrm{x}_{2}-\mathrm{x}_{1}\right)^{2}+\left(\mathrm{y}_{2}-\mathrm{y}_{1}\right)^{2}}$.

إن هذا الثرط طبيعي وجلي، لأنه إذا كانت التحويلات غير تقلصية، فإن النقاط في الصورة المستسخة

سوف تكون متباعدة، وسوف يترتب على ذلك أن تكون الصورة النهائية غير متتاهية الحجم. باستثناء هذا الشرط فإن التحويلات يمكن أن تأخذ أي صيغة، من الناحية العملية كما ذكرنا سابقا فإن التحويل يمكن أن يكون تآلفياً،

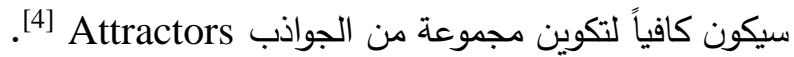

$w_{i}\left[\begin{array}{l}x \\ y\end{array}\right]=\left[\begin{array}{ll}a_{i} & b_{i} \\ c_{i} & d_{i}\end{array}\right]\left[\begin{array}{l}x \\ y\end{array}\right]+\left[\begin{array}{c}e_{i} \\ f_{i}\end{array}\right]$

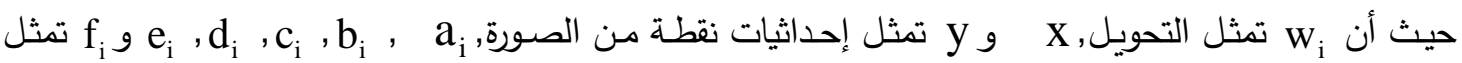




$$
\text { معاملات التحويل. }
$$

التحويلات التقلصية لها صفة مقنعة، وهي أن هذه التحويلات إذا طبقت مرارا وتكرارا، فإنها سوف تقترب من نقطة

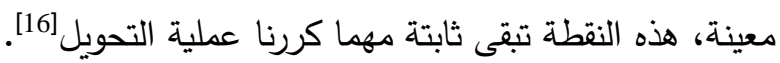

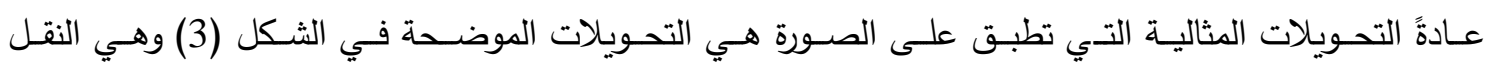
translation كل نقطة ضوئية في الثكل فضـلاً عن ذلك يمكن تطبيق التحويل على بعض النقاط الرئيسية التي تُعرف الثكل

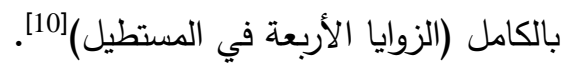
التعريف (1): نظام الدالة التكرارية:

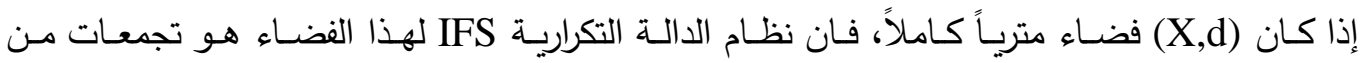
التطبيقات التقلصية ويمكن أن يعبر عنها بالثكل الآتي: $\mathrm{w}_{\mathrm{i}}=\mathrm{x} \rightarrow \mathrm{x}$, for $\mathrm{i}=1,2,3, \ldots, \mathrm{n}$

\section{التعريف (2): شرط ليبشتز :}

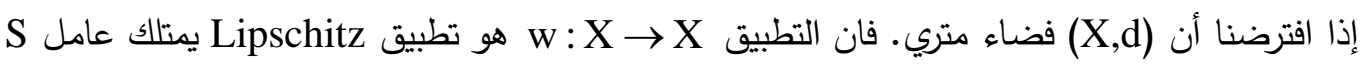
يسمى Lipschitz factor ، إذا وجدت قيمة حقيقية موجبة بحيث أن: $\mathrm{d}(\mathrm{w}(\mathrm{x}), \mathrm{w}(\mathrm{y})) \leq \mathrm{s} d(\mathrm{x}, \mathrm{y}), \forall \mathrm{x}, \mathrm{y} \in \mathrm{X}$.

إذا كان ثابت ليبثتز يحقق W تطبيق تقلصي بعامل التقليص S فان S S 7- المخطط العام لكبس الصور الرقمية لغرض كبس الصور الرقمية ذات التدرج الرمادي، يتم تقسيم الصورة إلى كتل المدى. وهناك كتل اكبر

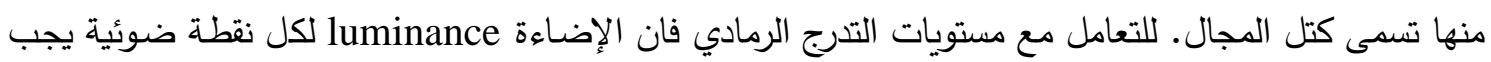
أن تقاس حسب المعادلة الآتية [2]: $\mathrm{Z}^{\prime}=\mathrm{s}_{\mathrm{i}} \mathrm{Z}+\mathrm{O}_{\mathrm{i}}$

باستخدام المعادلة السابقة نجد اقل خطأ تقريبي لكتلة المجال باستخدام التحويل التآلفي لتقليل mse، ويمكن حساب

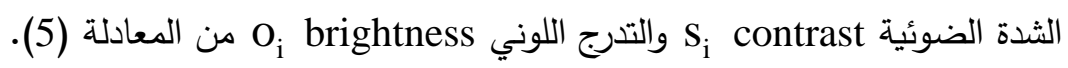
لتكن Z'=f'(x, y) قيمة النقطة الضوئية في كتلة المدى R ، وبما أن كتلة المجال حجمها اكبر بمرتين من كتلة المدى لذلك يكون من الضروري إجراء تحويل قياس لكتلة المجال باستخدام عوامل القياس وذلك بأخذ العينات

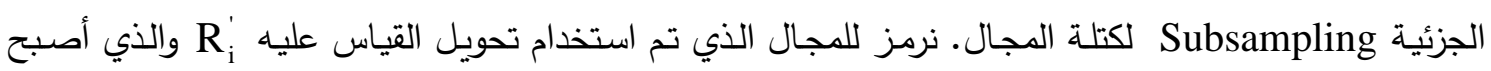
حجمه B×B.

بعدها يتم قياس المسـافة بين R و R وذلك باستخدام دالـة معدل مربـع الخطأ mse التي تعطى

بالمعادلة الآتية [6]؛ بلتص mse $=\frac{1}{N} \sum_{i=1}^{N}\left(R_{i}(x, y)-R_{i}^{\prime}(x, y)\right)^{2}$ 


$$
\begin{array}{r}
s_{i}=\frac{m \sum_{j=1}^{m} d_{i j} r_{i j}-\sum_{j=1}^{m} d_{i j} \sum_{j=1}^{m} r_{i j}}{m \sum_{j=1}^{m} d_{i j}{ }^{2}-\sum_{j=1}^{m}\left(d_{i j}\right)^{2}} \\
o_{i}=\frac{1}{m}\left[\sum_{j=1}^{m} r_{i j}-s_{i} \sum_{j=1}^{m} d_{i j}\right]
\end{array}
$$

لكل intensities النقاط الضوئية في كتلة المدى وكتلة المجال التي تحولت إلى كتلة مدى على التوالي وان m=B×B التي تمثل عدد النقاط الضوئية في كتلة المدى [16].

8- خوارزميات كبس الصور الرقمية باستخدام أنظمة الدالة التكرارية 1-8 الخوارزمية (1): خوارزمية كبس الصور ذات التدرج الرمادي باستخدام أنظمة الدالة التكرارية.
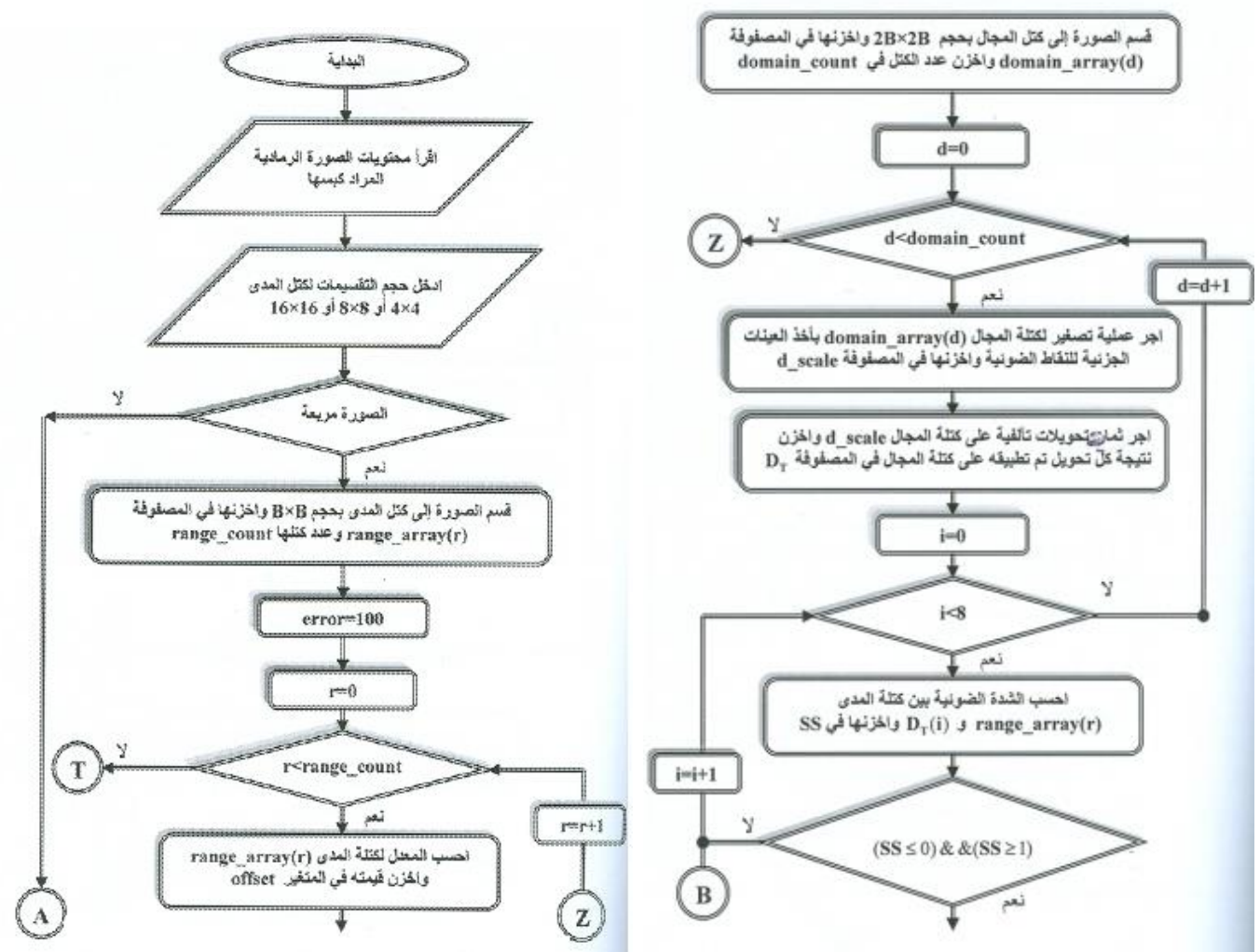


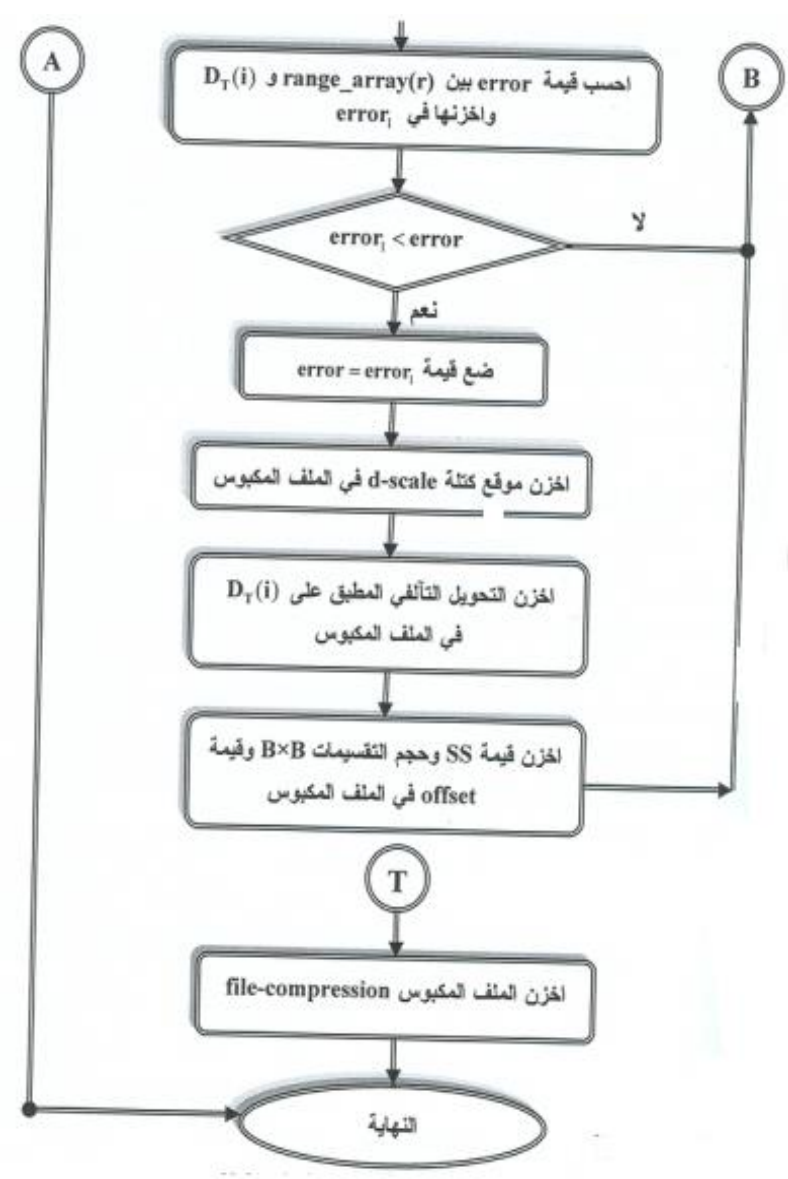

2-8 الخوارزمية (2): خوارزمية كبس الصور الملونة باستخدام أنظمة الدالة التكرارية

يتم تحليل الصـورة الرقميـة الملونـة (color image) إلى ثـلاث (شرائح) منفصلة وهي الصـورة ذات التدرجات الحمراء (Red image) والصورة ذات التدرجات الخضراء (Green image) والصورة ذات التدرجات

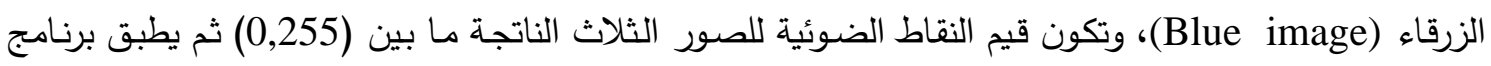

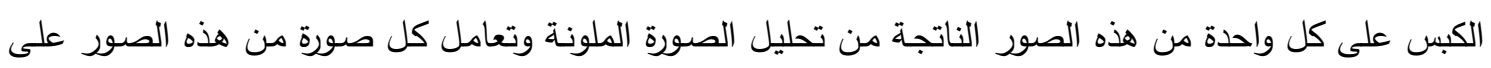
أنها صورة رمادية وتطبق عليها العمليات المذكورة في الخوارزمية (1) وبعد أن يتم كبس كل واحدة من هذه الثرائح يتم دمج الثرائح السابقة فيكون الناتج صورة ملونة علئة

Image Decompression فك الكبس للصور الرقمية

تعد عملية فك كبس المعلومات المخزونة في الملف الكسوري المكبوس اقل تعقيداً من عملية الكبس. إن عملية استرجاع الصورة من الصورة الككبوسة تتم بتكرار التحويل على صورة اختيارية لها نفس أبعاد الصورة الأصلية. 

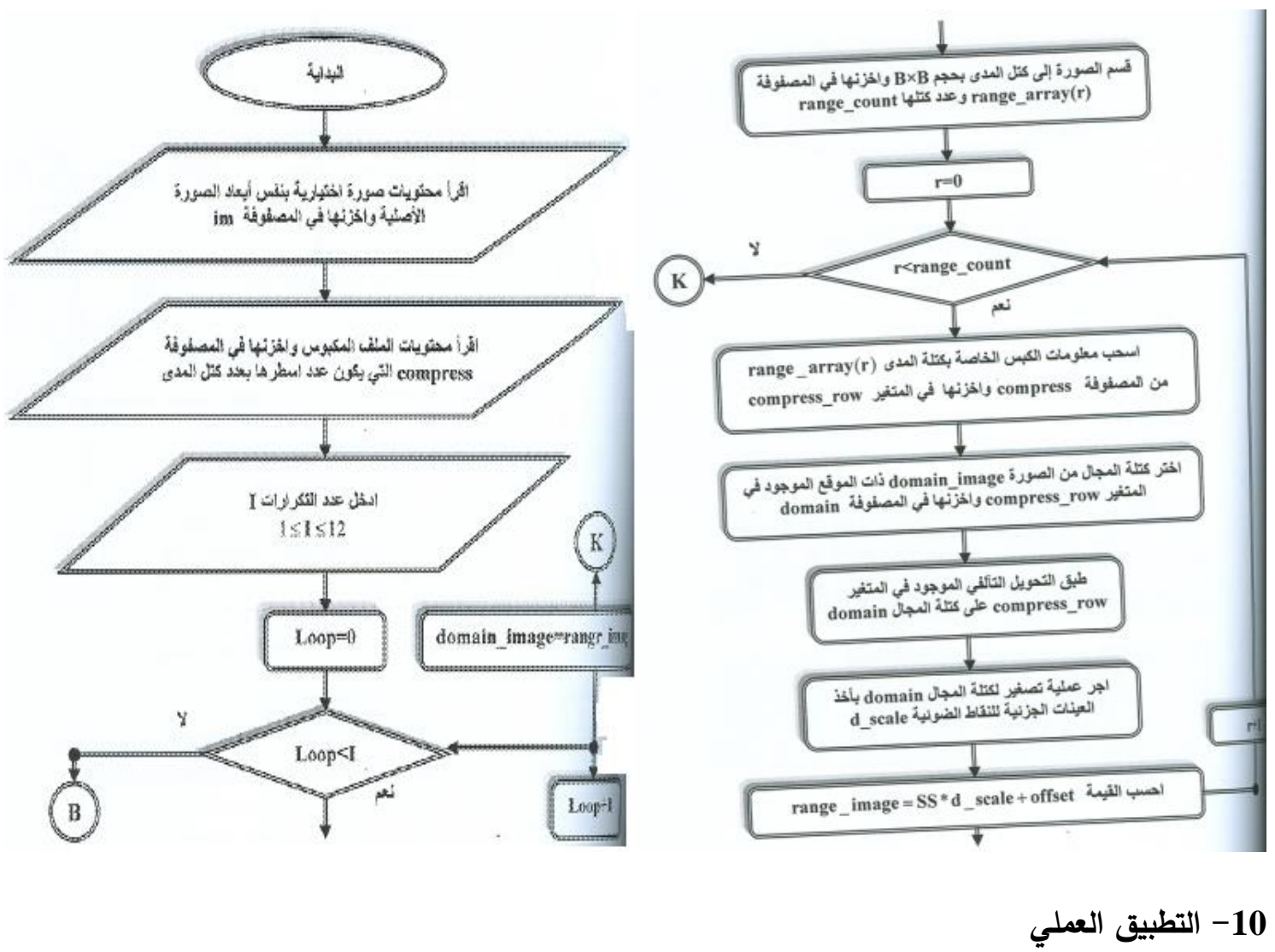

تم التطبيق العملي للخوارزميات السابقة على أربع وثلاثين عينة مختلفة لصور من مدينة الموصل ونظراً

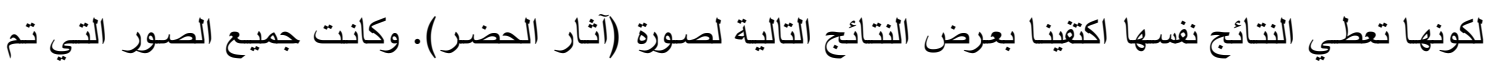

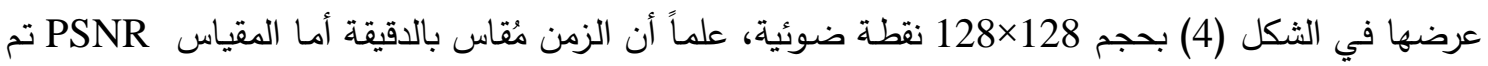

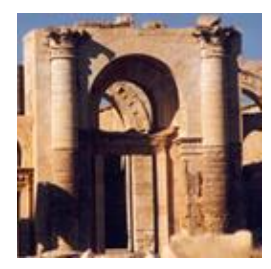

الصورة الرابعة

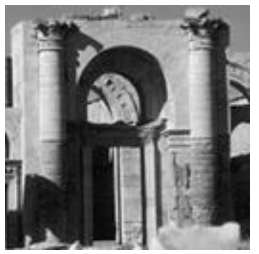

الصورة الرابعة

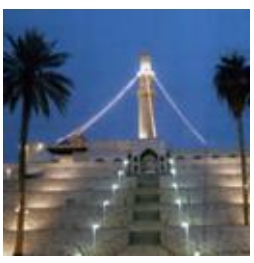

الصورة الثالثة

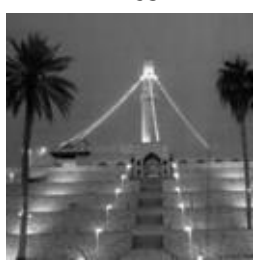

الصورة الثالثة

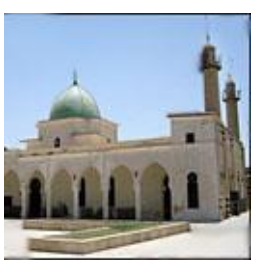

الصورة الثانية

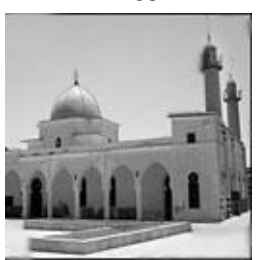

الصورة الثانية قياسه بوحدة (dB/ديسبل).

$$
\text { الثكل (4): الصور الرمادية والصور الملونة }
$$
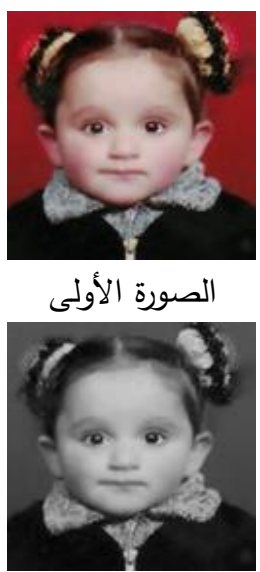

الصورة الأولى 


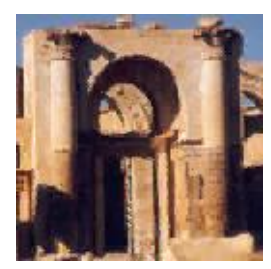

$-\dot{+}$

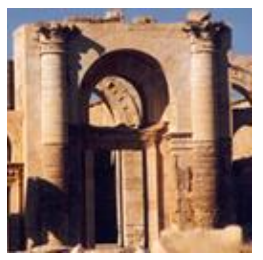

$-9$

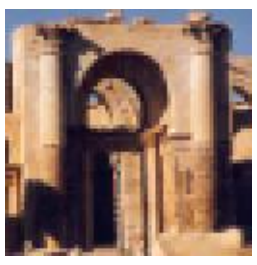

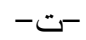

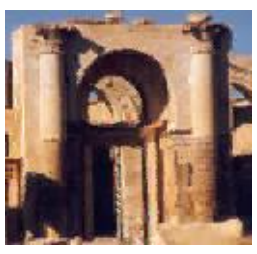

$-\rightarrow$

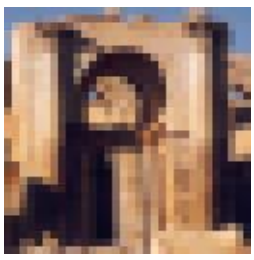

$-\underbrace{-}$

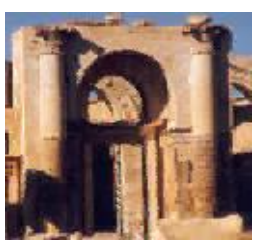

$-د$

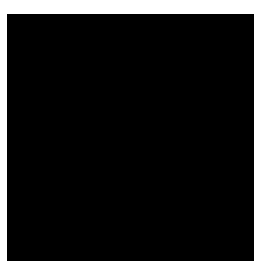

$-$

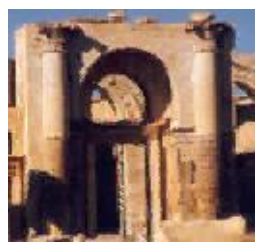

-

الثكل (5): -أ- صورة اختيارية، -ب- التكرار الأول، -ت- التكرار الثاني، -ث- التكرار الساد، -ج- التكرار الثامن، د- التكرار العاشر، -هـ- التكرار الثاني عشر (للتقسيمات بحجم 4×4) ، - - - الصورة الأصلية.

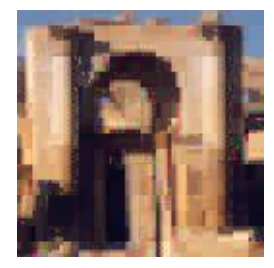

$-ث$

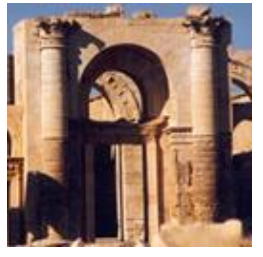

$-9$

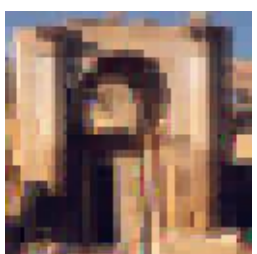

- -

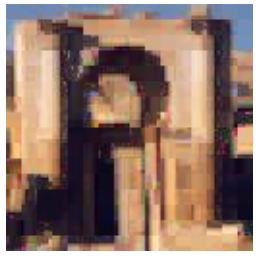

$-\infty$

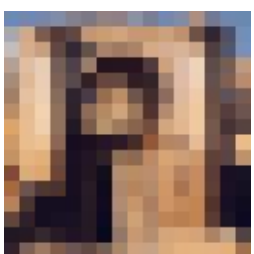

$-4$

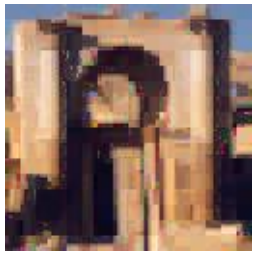

$-5$

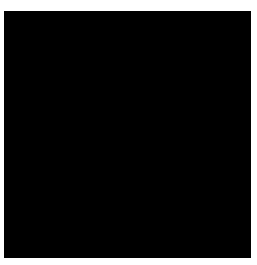

$-$

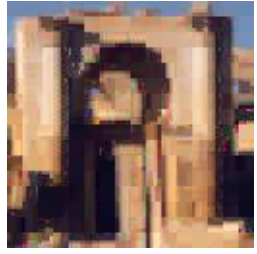

${ }^{-}$

الثكل (6): -أ- صورة اختيارية، -ب- التكرار الأول، -ت- التكرار الثاني، -ث- التكرار الساد، -ج- التكرار الثامن، د- التكرار العاشر، -هـ- التكرار الثاني عشر (للتقسيمات بحج 8×8) ، -و - الصورة الأصلية.

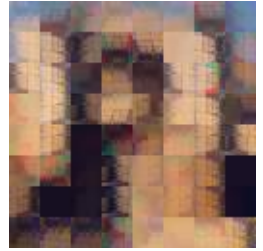

-ث

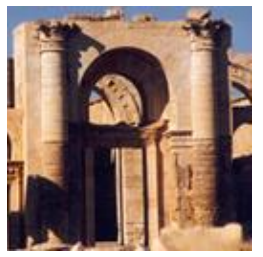

$-9$

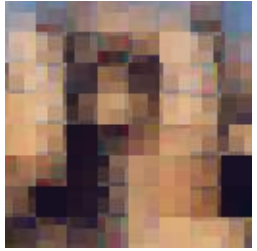

$-ت$

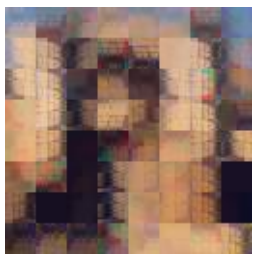

$-$

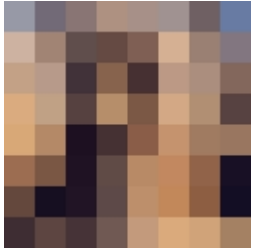

$-$

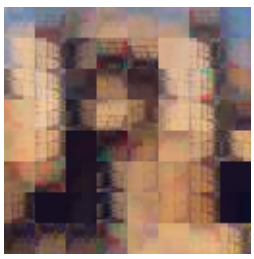

$-5$

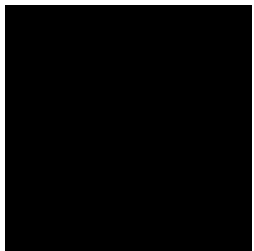

أ-

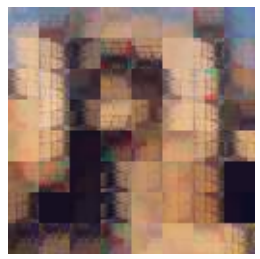

-

الثكل (7): -أ- صورة اختيارية، -ب- التكرار الأول، -ت- التكرار الثاني، -ث- التكرار الساد، -ج- التكرار الثامن، د- التكرار العاشر، -هـ- التكرار الثاني عشر (للتقسيمات بحجم 16×16) ، ـو - - - الصورة الأصلية. 
الجدول (1): نتائج التكرار الأول التي تم الحصول عليها من عملية فاك الكبس لصورة ملونة

بحجم 128×128 (آثار الحضر ).

\begin{tabular}{|c|c|c|c|}
\hline بحجم 16×16 بقات & 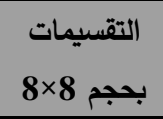 & بحجم 4×4 & المتفيرات \\
\hline 0.1388 & 1.6102 & 21.8458 & الوقت المستغرق لعملية الكبس \\
\hline 0.0042 & 0.0065 & 0.0177 & الوقت المستغرق لعملية فلك الكبس \\
\hline $1.27 \mathrm{~KB}$ & $3.2 \mathrm{~KB}$ & $11.2 \mathrm{~KB}$ & حجم الصورة بعد الكبس \\
\hline 8.1007 & 10.4454 & 13.1934 & SNR_ قيمة \\
\hline $27.8943 \mathrm{ds}$ & $29.0734 \mathrm{ds}$ & $30.3385 \mathrm{ds}$ & قيمة PSNR \\
\hline
\end{tabular}

الجدول(2): نتائج التكرار الرابع التي تم الحصول عليها من عملية فلك الكبس لصورة ملونة بحجم 128×128.

\begin{tabular}{|c|c|c|c|}
\hline بحجم 16×16 باتقسيمات & بحجم 8×8 8 & 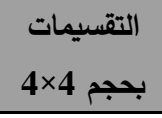 & المتغيرات \\
\hline 0.1388 & 1.6102 & 21.8458 & الوقت المستغرق لعملية الكبس \\
\hline 0.0120 & 0.0203 & 0.0521 & الوقت المستغرق لعملية فلك الكبس \\
\hline $1.27 \mathrm{~KB}$ & $3.2 \mathrm{~KB}$ & $11.2 \mathrm{~KB}$ & حجم الصورة بعد الكبس \\
\hline 10.2216 & 13.5625 & 18.0932 & قيمةSNR \\
\hline $28.5026 \mathrm{ds}$ & $30.5569 \mathrm{ds}$ & $32.5451 \mathrm{ds}$ & قيمة PSNR \\
\hline
\end{tabular}

الجدول (3): نتائج التكرار الثامن التي تم الحصول عليها من عملية فك الكبس لصورة ملونة بحجم 128×128.

\begin{tabular}{|c|c|c|c|}
\hline بحجم 16×16 بقات 16 & بحجم 8×8 التقسيمات & بحجم 4×4 التقسيمات & المتغيرات \\
\hline 0.1388 & 1.6102 & 21.8458 & الوقت المستغرق لعملية الكبس \\
\hline 0.0159 & 0.0404 & 0.1250 & الوقت المستغرق لعملية فك الكبس \\
\hline $1.27 \mathrm{~KB}$ & $3.2 \mathrm{~KB}$ & $11.2 \mathrm{~KB}$ & حجم الصورة بعد الكبس \\
\hline 10.3068 & 13.9906 & 18.1049 & قيمة \\
\hline $28.5488 \mathrm{ds}$ & $30.4389 \mathrm{ds}$ & $32.6641 \mathrm{ds}$ & قيمة PSNR \\
\hline
\end{tabular}

الجدول (4): نتائج التكرار الثاني عشر التي تم الحصول عليها من عملية فك الكبس لصورة ملونة بحم 128×128.

\begin{tabular}{|c|c|c|c|}
\hline بحم 16×16 التقسيمات & بحم 8×8 التقيمات 8 & بحجم 4×4 & المتغيرات \\
\hline 0.1388 & 1.6102 & 21.8458 & الوقت المستغرق لعملية الكبس \\
\hline 0.0203 & 0.0596 & 0.1781 & الوقت المستغرق لعملية فلك الكبس \\
\hline $1.27 \mathrm{~KB}$ & $3.2 \mathrm{~KB}$ & $11.2 \mathrm{~KB}$ & حجم الصورة بعد الكبس \\
\hline 10.3083 & 13.9999 & 18.0724 & قيمة SNR \\
\hline $28.5478 \mathrm{ds}$ & $30.4222 \mathrm{ds}$ & $32.6460 \mathrm{ds}$ & قيمة PSNR \\
\hline
\end{tabular}




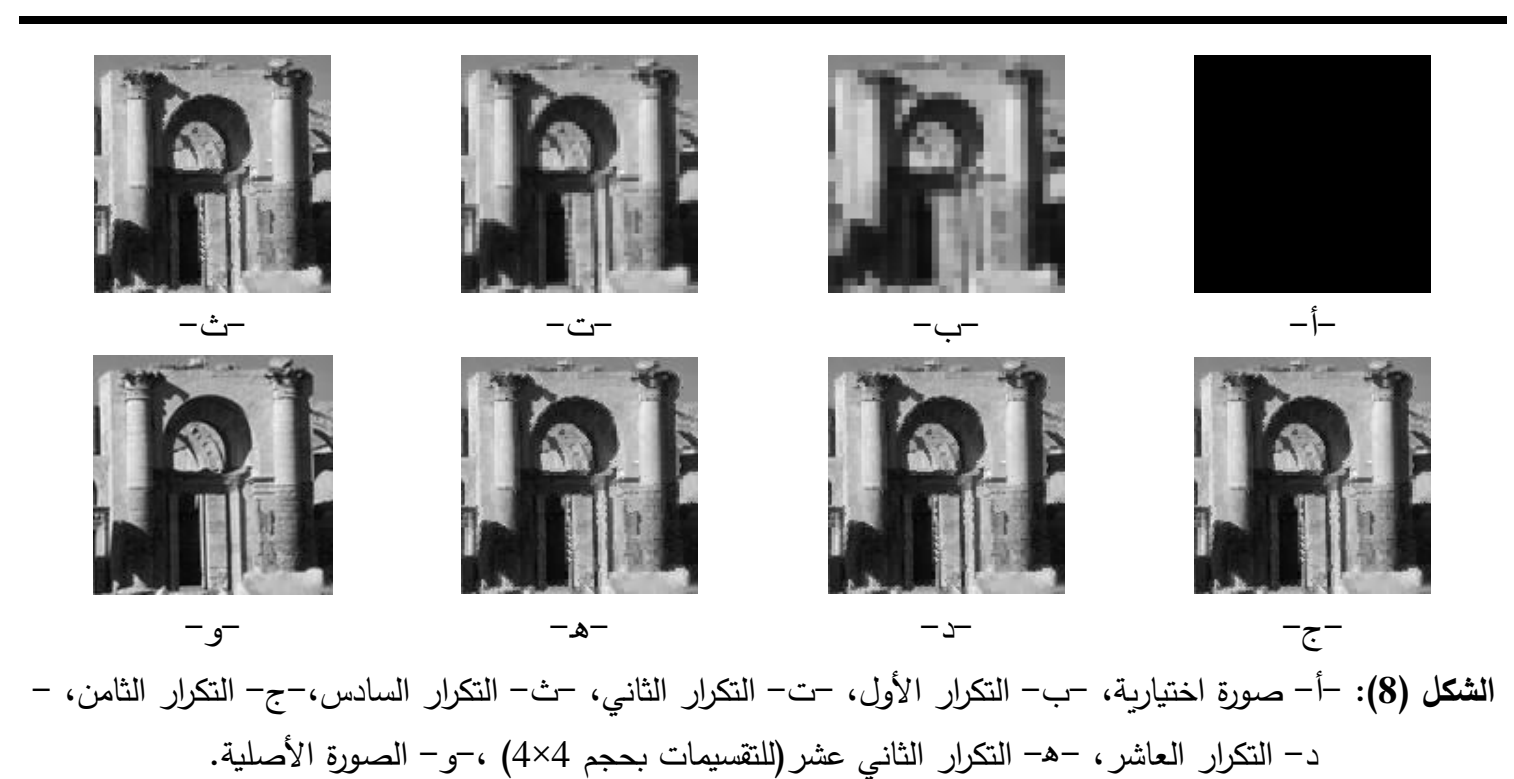

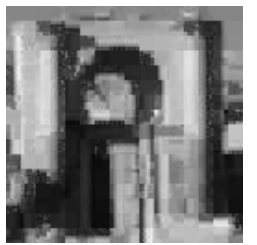

$-\stackrel{+}{-}$

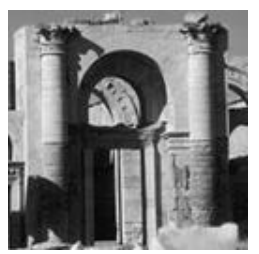

-

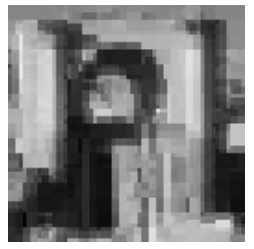

- -

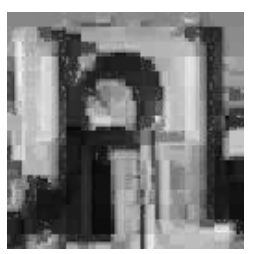

$-\infty$

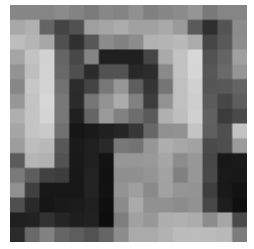

-ب

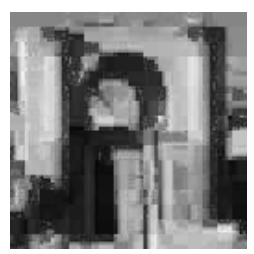

$-5$

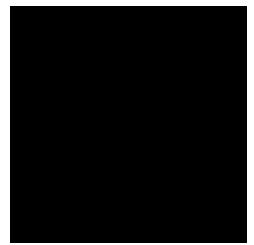

$-\mathfrak{-}-$

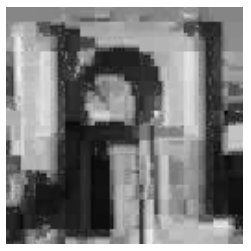

-

الثكل (9): -أ- صورة اختيارية، -ب- التكرار الأول، -ت- التكرار الثاني، -ث- التكرار الساد، -ج- التكرار الثامن، د- التكرار العاشر، - ه- التكرار الثاني عشر (للتقسيمات بحجم 8×8) ، -و - الصورة الأصلية.

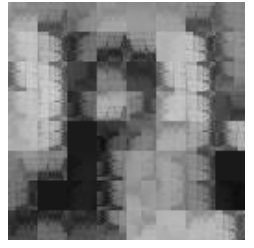

$-\stackrel{+}{-}$

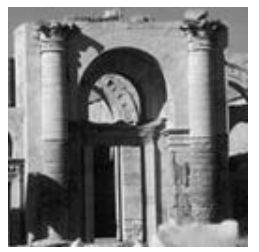

$-9$

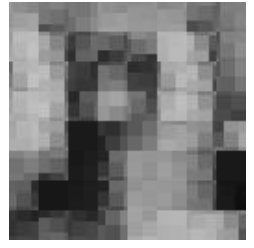

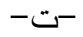

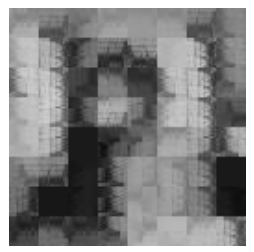

$-\infty$

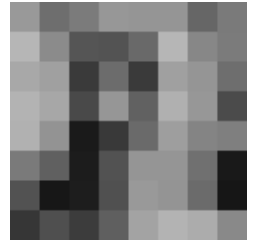

-ب

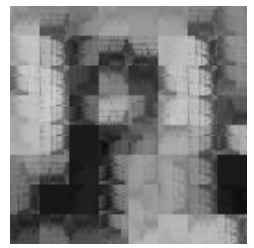

$-2$

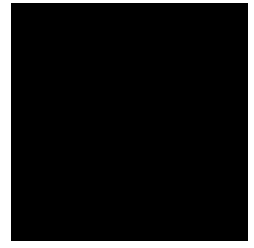

$-1-$

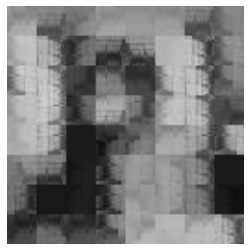

-

الثكل (10): -أ- صورة اختيارية، -ب- التكرار الأول، -ت- التكرار الثاني، -ث- التكرار الساد، -ج- التكرار الثامن، - - - التكرار العاشر ، -ه- التكرار الثاني عشر (للتقسيمات بحجم 16×16) ، -و - الصورة الأصلية. 
الجدول (5): نتائج التكرار الأول التي تم الحصول عليها من عملية فاك الكبس لصورة رمادية

بحجم 128×128 1 (آثار الحضر)

\begin{tabular}{|c|c|c|c|}
\hline بحجم 16×التقسيمات 16 & بحجم 8×8 & بحجم 4×4 التقسيمات & المتغيرات \\
\hline 0.0487 & 0.5589 & 7.5068 & الوقت المستغرق لعملية للكبس \\
\hline 0.0018 & 0.0031 & 0.0065 & الوقت المستغرق لعملية فك الكبس \\
\hline 752 bytes & $1.38 \mathrm{~KB}$ & $4.09 \mathrm{~KB}$ & حجم الصورة بعد الكبس \\
\hline 8.2347 & 10.5487 & 13.2785 & قيمةSNR \\
\hline $27.6315 \mathrm{ds}$ & $28.9313 \mathrm{ds}$ & $30.2265 \mathrm{ds}$ & قيمة PSNR \\
\hline
\end{tabular}

الجدول (6): نتائج التكرار الرابع التي تم الحصول عليها من عملية فك الكبس لصورة رمادية بحجم 128×128.

\begin{tabular}{|c|c|c|c|}
\hline بحجم 16×16 بقات & بحجم 8×8 & بحجم 4×4 & المتغيرات \\
\hline 0.0487 & 0.5589 & 7.5068 & الوقت المستغرق لعملية للكبس \\
\hline 0.0031 & 0.0065 & 0.0180 & الوقت المستغرق لعملية فك الكبس \\
\hline 752 bytes & $1.38 \mathrm{~KB}$ & $4.09 \mathrm{~KB}$ & حجم الصورة بعد الكبس \\
\hline 10.3210 & 13.6543 & 18.1710 & قيمة SNR \\
\hline $28.3845 \mathrm{ds}$ & $30.4175 \mathrm{ds}$ & $32.6223 \mathrm{ds}$ & قيمة PSNR \\
\hline
\end{tabular}

الجدول (7): نتائج التكرار الثامن التي تم الحصول عليها من عملية فاك الكبس لصورة رمادية بحجم 128×128.

\begin{tabular}{|c|c|c|c|}
\hline بحجم 16×16 التقسيمات & بحجم 8×8 8 & 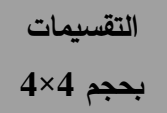 & المتغيرات \\
\hline 0.0487 & 0.5589 & 7.5068 & الوقت المستغرق لعملية للكبس \\
\hline 0.0049 & 0.0148 & 0.0437 & الوقت المستغرق لعملية فك الكبس \\
\hline 752 bytes & $1.38 \mathrm{~KB}$ & $4.09 \mathrm{~KB}$ & حجم الصورة بعد الكبس \\
\hline 10.3948 & 14.0874 & 18.2311 & SNR \\
\hline $28.4262 \mathrm{ds}$ & $30.2829 \mathrm{ds}$ & $32.7513 \mathrm{ds}$ & قيمة PSNR \\
\hline
\end{tabular}

الجدول (8): نتائج التكرار الثاني عشر التي تم الحصول عليها عملية فاك الكبس لصورة رمادية بحجم 128×128.

\begin{tabular}{|c|c|c|c|}
\hline بحجم 16×16 بلتصيمات & بحجم 8×8 & بحجم 4×4 & المتغيرات \\
\hline 0.0487 & 0.5589 & 7.5068 & الوقت المستغرق لعملية للكبس \\
\hline 0.0086 & 0.0201 & 0.0510 & الوقت المستغرق لعملية فك الكبس \\
\hline 752 bytes & $1.38 \mathrm{~KB}$ & $4.09 \mathrm{~KB}$ & حجم الصورة بعد الكبس \\
\hline 10.3968 & 14.0783 & 18.2270 & قيمة SNR \\
\hline $28.4249 \mathrm{ds}$ & $30.2490 \mathrm{ds}$ & $32.7561 \mathrm{ds}$ & قيمة PSNR \\
\hline
\end{tabular}


من خـلال النتائج التي تم الحصول عليها يتبين بـان أفضل وضوحية للصورة المسترجعة التي تكون تقسيماتها بحجم 4×4 للتكرار الثاني عشر وذلك لأنها تعطي نتائج كبس جيدة بالنسبة لبقية التكرارات للتقسيمات ذات الحجم 8×8 و16×16 وتبعا لذلك تكون النتائج التي تم الحصول عليها باستخدام أربعة معايير لقياس نوعية الصورة الناتجة من عملية الكبس. وبما أن طريقة الكبس باستخدام أنظمة الدالة التكرارية هي من طرائق الكبس بالفقدان لذلك تمتاز هذه

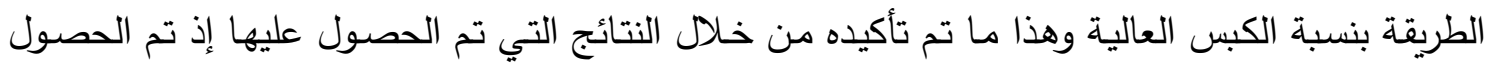
على نسبة جيدة للكبس ونوعية جيدة وواضحة. وهنالك عدة عوامل تؤثر على عملية الكبس وهذه العوامل هي: هيده وونه

1-11 علاقة الزمن بحجم الصورة:

يقصد بالزمن الوقت المستغرق لعملية كبس الصسورة وعملية استرجاعها من الملف المكبوس علما أن الزمن مقاس بالاقيقة. إن زيادة حجم الصورة يعني زيادة في مساحتها مما يؤدي إلى زيادة عدد الكتل التي يتت مطابقتها ويؤدي إلى زيادة في كل من زمن الكبس وزمن الاسترجاع للصورة وكما هو واضح في الثكل (11).

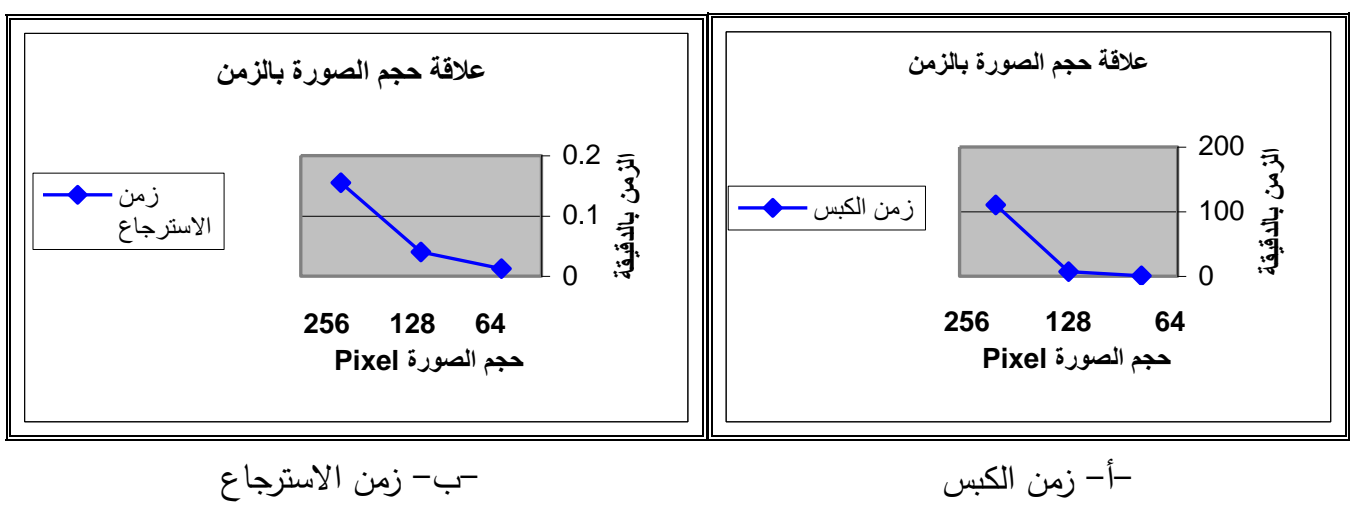

الثكل (11): تأثير حجم الصورة على الزمن.

2-11 علاقة الزمن بعدد التكرارات:

يقصد بعدد التكرارات عدد مرات استرجاع الصورة من الملف المكبوس لحين الحصول على صورة تكون اقرب ما يمكن من الصورة الأصلية. وتبين أن زمن الكبس لا يتأثر بزيادة عدد التكرارات، في الوقت نفستهِ يزداد زمن الاسترجاع لزيادة عدد المربعات التي يتم مطابقتها في كل تكرار وهذا ما يبينه الثكل (12). 

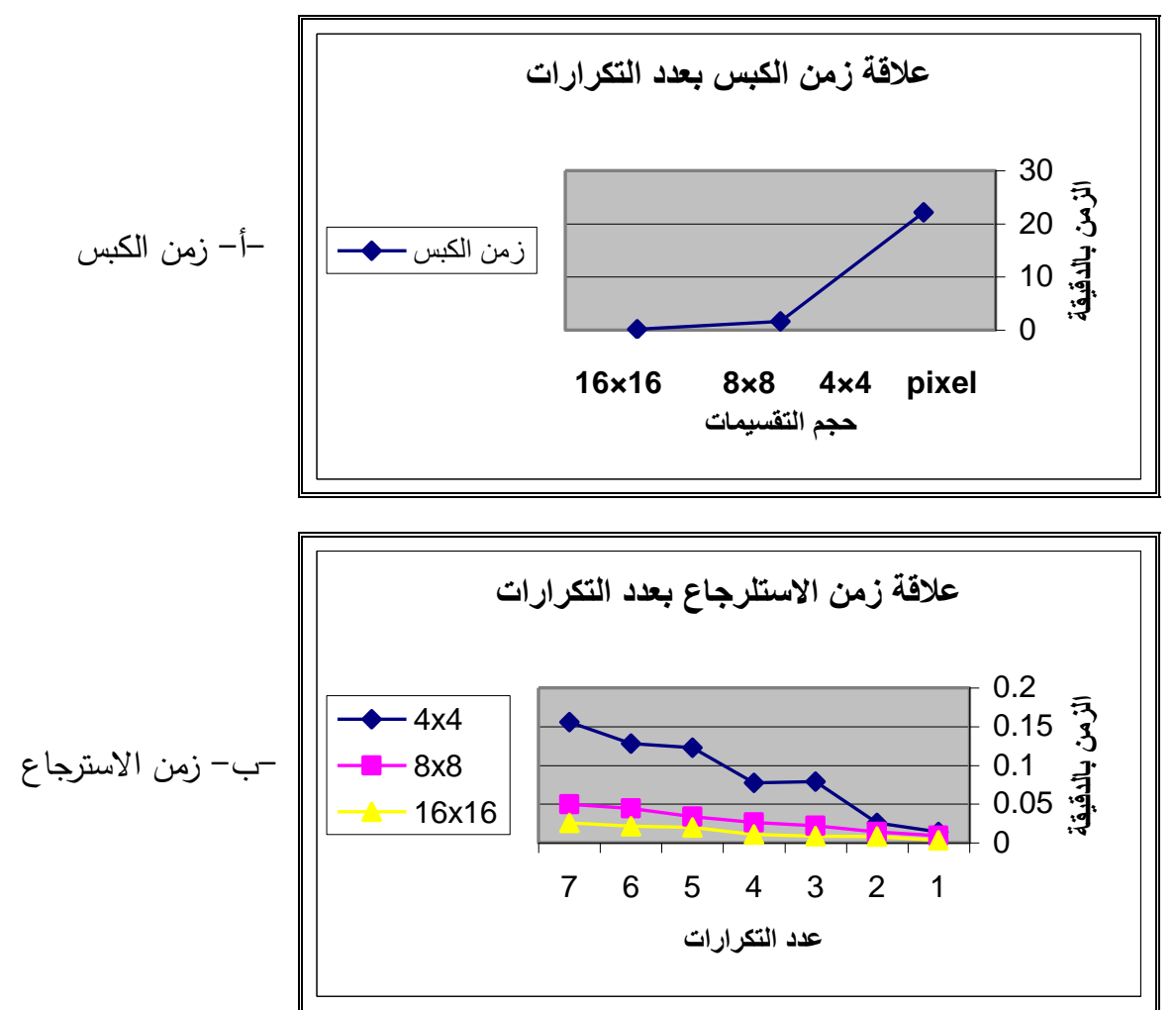

الثكل (12): تأثير الزمن على عدد التكرارات.

3-11 علاقة حجم التقسيمات بنسبة ذروة الإثارة إلى الضوضاء

نسبة الإشارة إلى الضوضاء PSNR (تم قياسها بوحدة dB) يمثل مقياساً لوغارتميا لمعدل مربع الخطأ

بين الصورة المسترجعة A(x,y) من عملية الكبس وبين الصورة الأصلية f(x,y)، تكون الصور أفضل عندما

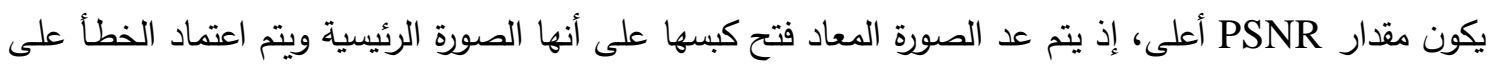
انه الضوضاء. وتبين انه كلما زادت قيمة PSNR زادت وضوحية الصورة المسترجعة مع زيادة عدد التكرارات وذلك

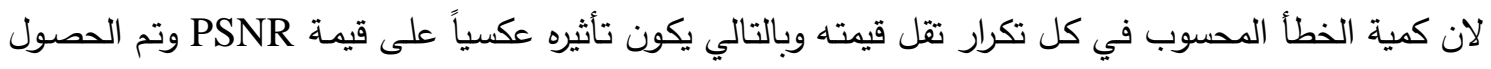
على النتيجة نفسها للمقياس SNR كما هو مبين في الثكل (13).

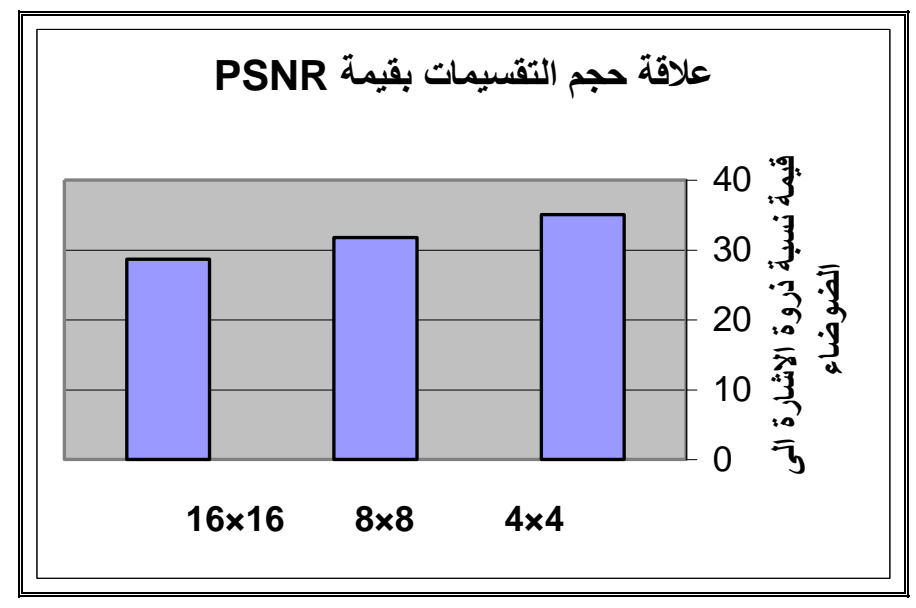

الثكل (13): تأثير حجم التقسيمات على قيمة PSNR. 
e e eلاقة عدد التكرارات بجذر معدل مربع الخطأ 4-11

تبين أنه في حأدي الحصول على قيمة صغيرة في مصفوفة الخطأ أي أن الصورة

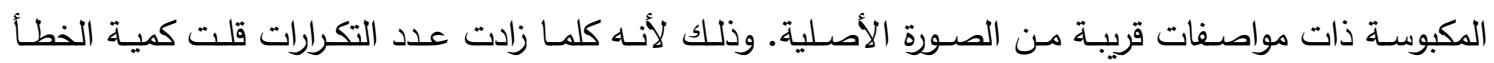
المحسوب حسب المعادلة (5) وكما هو مبين في الثكل (14).

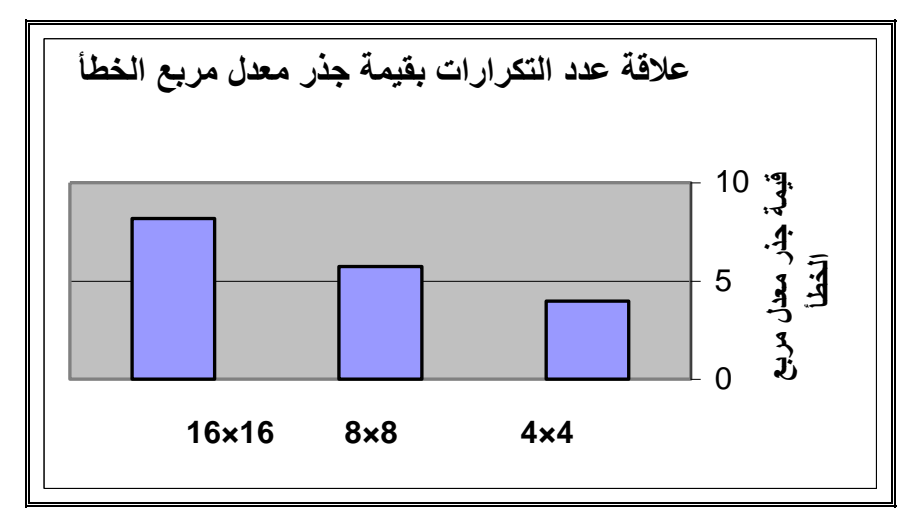

الثكل (14): تأثير عدد التكرارات على قيمة جذر معدل مربع الخطأ.

والنتائج الددونة في الجدولين (9) و (10) تم حسابها لتقسيمات المدى بحجم 4×4 في التكرار الثاني عشر لكونه اقرب للصورة الأصلية.

الجدول (9): النتائج العملية للتطبيق على صور ملونة بحجم 128×128 .

\begin{tabular}{|c|c|c|c|c|c|}
\hline $\mathbf{e}_{\mathrm{rms}}$ & PSNR & SNR & وقت فلك الكبس & وقت الكبس & اسم الصورة \\
\hline 3.9634 & 35.0435 & 21.8239 & 0.1555 & 22.1164 & صورة 1 \\
\hline 6.0490 & 32.4971 & 18.0479 & 0.1844 & 20.0927 & صورة 2 \\
\hline 4.7387 & 34.6176 & 19.5128 & 0.1589 & 20.7258 & صورة 3 \\
\hline 5.9462 & 32.6460 & 18.0724 & 0.1781 & 21.8458 & صورة 4 \\
\hline
\end{tabular}

الجدول (10): النتائج العملية للتطبيق على صور ذات تدرج رمادي بحجم 128×128.

\begin{tabular}{|c|c|c|c|c|c|}
\hline $\mathbf{e}_{\mathrm{rms}}$ & PSNR & SNR & وقت فلك الكبس & وقت الكبس & اسم الصورة \\
\hline 3.8685 & 34.5686 & 21.9333 & 0.0526 & 7.3297 & صورة 1 \\
\hline 6.0968 & 32.4287 & 18.1730 & 0.0516 & 6.0716 & صورة 2 \\
\hline 4.4776 & 35.0415 & 19.3695 & 0.0534 & 6.9378 & صورة 3 \\
\hline 5.8713 & 32.7561 & 18.2270 & 0.0510 & 7.5068 & صورة 4 \\
\hline
\end{tabular}

وبما أن طريقة الكبس باستخدام أنظمة الدالة التكرارية هي من طرائق الكبس بالفقدان لذلك تمتاز هذه

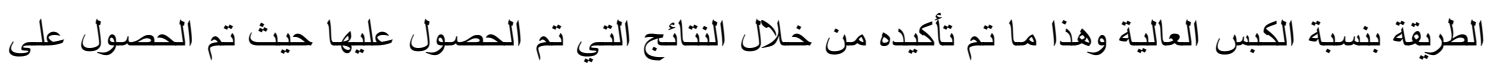
نسبة جيدة للكبس ونوعية جيدة وواضحة. يمكن حساب نسبة الكبس بالطريقة الآتية: نسبة الكبس (Compression Ratio) = $=$ 
الجدول (11): نسبة الكبس للصور الملونة بحجم 128×128.

\begin{tabular}{|c|c|c|c|c|}
\hline الصورة الرابعة & الصورة الثالثة & الصورة الثانية & الصورة الأولى & سم الصورة \\
\hline 4.2857 & 4.4037 & 4.5283 & 4.4037 & نسبة الكبس \\
\hline
\end{tabular}

الجدول (12): نسبة الكبس للصور الرمادية بحجم 128×128.

\begin{tabular}{|c|c|c|c|c|}
\hline الصورة الرابعة & الصورة الثالثة & الصورة الثانية & الصورة الأولى & اسم الصورة \\
\hline 4.1565 & 4.3478 & 4.3814 & 4.2607 & نسبة الكبس \\
\hline
\end{tabular}

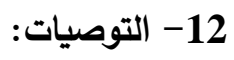

إن ما تم بناؤه من برمجيات يمكن أن يكون نقطة انطلاق لأفكار وأعمال مستقبلية كما يأتي:

\& يمكن استخدم إحدى تقنيات الكبس لتطوير كفاءة البرنامج لكبس الصور الرقدية.

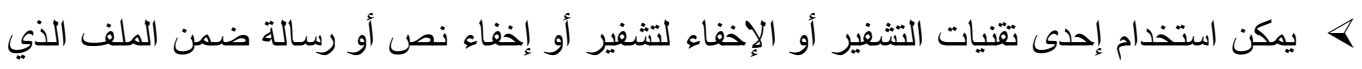
يتم كبسه باستخدام أنظمة الدالة التكرارية لكون الملف الذي يتم كبسه صغير الإه الحجم. 


$$
\text { الخياط، باسل يونس ذنون، "مدخل إلى النمذجة الرياضية باستخدام الـ } 2011 \text { (قيد الطباعة). }
$$

[2] Conci, Aura and A quino, Feliper (2005), "Fractal coding based on image local fractal dimension", Fluminense University, Brazil. Volume 24, N. 1, pp. 83-98.

[3] Edgar, Gerald and Axler, S. and Ribet, K.A. (2008), "Measure, Topology, and Fractal Geometry", Springer, University of California, USA.

[4] El-Khamy, Said E., Abdou, Hossam El-Din .M (1992), "Anovel secure image coding scheme using fractal transformation", Alexandria University, Egypt.

[5] Fisher, Yuval (1995), "Fractal Image Compression Theory and Application", Springer-Verlag, New York.

[6] Hart, John C., (1995), "Fractal image compression and the inverse problem of recurrent iterated function systems", Washington State University, USA.

[7] Hassaballah, M., Makky. M. M and Mahdy, Youssef. B, (2005), "A Fast fractal image compression method based entropy", vol. 1, pp.30-40, 2005, South Valley University, Egypt.

[8] Jacquin, Arnaud E., (1992), "Image coding based on a fractal theory of iterated contractive image transformations", IEEE Transactions on Image Processing, vol. 1, no. 1, 1992.

[9] Lynch, Stephen (2001), "Dynamical Systems with Applications using MAPLE", Birkhäuser .

[10] Maharatna, K. and Banerjee, Swapna (2002), "Cordic based array archttecture for affine transformation of images", Indian Institute of Technology.

[11] Paus, Petr (2006), "Computer analysis of fractal sets", Czech-Technical University, Prague, 14-17, pp. 172-176.

[12] Salomon, David (2007), "Data Compression: Fourth Edition", Springer-Verlag, London, U.K.

[13] Sankaranarayanan, Viswanath (1998), "Fractal image compression literature survey".

[14] Suman, Mitra K., C, A, Murthy, Malay K, Kundu and Bhargab B. Bhattacharya (2001), "Fractal image compression using iterated function system with probabilities", Indian Statistical Institute IEEE Transformation on Image Process.

[15] Umbaugh, Scotte E., (1998), "Computer Vision and image Processing a practical approach using CVIP Tools", Prentice Hall.

[16] Xiao, Ke, (2004), "Fractal compression and analysis on remotely sensed imagery", Unpublished Ph. D Thesis Louisiana State University.

[17] Young, Ian T., Gerbrands, Jan J. and Vliet, Lucas J. van (1998), "Fundamentals of Image Processing", Delft University of Technology, Netherlands. 